\title{
A COMPREENSÃO DA VELHICE E DO ENVELHECER NA VOZ DOS PROFISSIONAIS DE SAÚDE DA FAMÍLIA
}

\author{
Camila Amthauer \\ João Werner Falk²
}

resumo

A velhice é um processo complexo de alterações na trajetória de vida das pessoas. Refletir acerca da representatividade do envelhecimento é um caminho para compreender o real significado de envelhecer, permitindo aos profissionais de saúde o planejamento de estratégias fundamentadas na realidade. O objetivo deste trabalho é conhecer o que os profissionais atuantes na Estratégia de Saúde da Família (ESF) atribuem ao envelhecimento e sua percepção acerca desse processo. Trata-se de um recorte da pesquisa intitulada "O cuidado à pessoa idosa na ótica de profissionais da Estratégia de Saúde da Família". A pesquisa é um estudo descritivo, de natureza qualitativa, desenvolvido junto aos profissionais da ESF de uma Unidade Básica

1 Enfermeira graduada pela Universidade Federal de Santa Maria (UFSM/CESNORS). Especialista em Saúde Pública pela Universidade Federal do Rio Grande do Sul (UFRGS). Mestranda no Programa de Pós-Graduação em Enfermagem pela UFRGS. E-mail: camila.amthauer@hotmail.com.

2 Médico graduado pela Universidade Federal do Rio Grande do Sul (UFRGS). Doutor em Ciências Médicas pela UFRGS. Professor da Faculdade de Medicina UFRGS. E-mail: joaofalk@terra.com.br. 
de Saúde de Porto Alegre/RS. A amostra foi aleatória e constituiu-se de 16 profissionais (quatro médicos, quatro enfermeiros, quatro técnicos de enfermagem e quatro agentes comunitários de saúde). A coleta de dados ocorreu no mês de dezembro de 2013, por meio de entrevistas semiestruturadas. A análise dos dados foi baseada na análise de conteúdo temática, proposta por Minayo. Quando os participantes foram questionados acerca de sua concepção sobre o processo de envelhecer, emergiram diferentes percepções, agrupadas nas seguintes categorias: processo natural do ser humano; autonomia e qualidade de vida; o jeito antigo e o novo; as perdas. $\bigcirc$ envelhecimento traz transformações para a sociedade. Para atender essa demanda e os paradigmas acerca do envelhecimento é preciso que os profissionais atendam o idoso em sua totalidade. Há necessidade de compreender o idoso e interagir com ele para que haja resolutividade nas ações realizadas pela equipe de saúde.

palavras-chave

Saúde da Família. Saúde do idoso. Envelhecimento. Profissional da saúde.

\section{Introdução}

O aumento da população acima de sessenta anos gera transformações nos valores e no modo como se percebe o processo de envelhecimento, havendo a necessidade de uma mudança no atendimento ao idoso, visto que o envelhecimento populacional traz novas necessidades e questões fundamentais para os profissionais que cuidam da população idosa. É preciso reconhecer, na sua totalidade, que o idoso carece de cuidados individualizados e personalizados, em função dos eventos específicos que o acometem durante a vida (HAMMERSCHMIDT; LENARDT, 2010).

A velhice é um processo complexo de alterações na trajetória de vida das pessoas. Cada contexto tem suas particularidades que vão alterar o estilo de vida de cada um. Os modos de revelar o significado da velhice e o processo de envelhecer para o idoso dependerão de como viveu e fez suas adaptações e enfrentamentos cotidianos. A repercussão do envelhecer vai depender da história de vida pessoal, da disponibilidade de suporte afetivo, das redes sociais, do sistema de valores pessoais e do estilo de vida adotado por cada um (FREITAS; QUEIROZ; SOUSA, 2010). 
Existe uma diversidade de questionamentos quando pensamos na representação do "ser velho" e dos contextos que determinam a velhice, podendo ser analisados os fatores que propiciam a classificação e o reconhecimento como velho que, em sua maioria, denota uma visão preconceituosa. Essa visão sobre o processo de envelhecimento, por vezes, decorre da informação equivocada, gerando significados e imagens negativas, comprometendo a vivência e a interação entre as pessoas. Os aspectos culturais também interferem na maneira de olhar o envelhecimento e como o idoso vai se constituir nesse meio (GUERRA; CALDAS, 2010).

Refletir acerca da representatividade do envelhecimento é um caminho para compreender o real significado de envelhecer, permitindo aos profissionais de saúde o planejamento de estratégias fundamentadas na realidade, que permitam proporcionar a manutenção da autonomia e independência do idoso, tendo como parâmetro a compreensão das alterações decorrentes do envelhecimento, possibilitando a melhoria da qualidade de vida consoante às condições de saúde em que o idoso se encontra (FREITAS; QUEIROZ; SOUSA, 2010).

A longevidade está ocupando um espaço significativo na sociedade, levando a população a adaptar-se com essa nova realidade. Os idosos representam um grupo diferenciado entre si e aos demais grupos, o que requer uma atenção especial em relação às características bastante peculiares dessa faixa etária, o que exige dos profissionais de saúde um novo olhar no cuidado do idoso, que vai além de um conjunto de habilidades clínicas e técnicas (FERREIRA et al., 2010; GARBIN et al., 2010).

Assim, neste estudo, buscamos conhecer o que os profissionais atuantes em uma Estratégia de Saúde da Família atribuem ao processo de envelhecimento e sua percepção acerca do envelhecer. Acreditamos que, com os resultados obtidos, os profissionais de saúde possam qualificar sua assistência e oferecer ao idoso um atendimento baseado em suas reais necessidades de saúde, considerando as peculiaridades que o processo de envelhecer traz para o idoso.

\section{Metodologia}

Trata-se de um recorte da pesquisa intitulada "O cuidado à pessoa idosa na ótica de profissionais da Estratégia de Saúde da Família", considerando-se o objeto de estudo, que se sintetiza na indagação: "qual a concepção de cuidado e práticas a serem desenvolvidas junto aos idosos usuários de uma Unidade Básica de Saúde, na ótica de profissionais atuantes na Estratégia de Saúde da Família no município de Porto Alegre/RS?". 
A pesquisa é um estudo descritivo, de natureza qualitativa, desenvolvido junto aos profissionais da Estratégia de Saúde da Família (ESF) atuantes em uma Unidade Básica de Saúde, pertencente ao Hospital de Clínicas de Porto Alegre (HCPA), um hospital universitário, vinculado à Universidade Federal do Rio Grande do Sul (UFRGS), no município de Porto Alegre, Rio Grande do Sul.

A amostra foi aleatória e constituiu-se de 16 profissionais (quatro médicos, quatro enfermeiros, quatro técnicos de enfermagem e quatro agentes comunitários de saúde). A determinação do número de sujeitos no estudo, respeitando o critério de representatividade profissional e das equipes, deu-se por saturação dos dados, considerando-se que a amostra ideal é aquela que reflete a totalidade em suas múltiplas dimensões, sendo suficiente o número que permita certa reincidência de informações (MINAYO, 2010).

Todos os convidados aceitaram participar do estudo e assinaram o Termo de Consentimento Livre e Esclarecido. O projeto de pesquisa foi aprovado pelo Comitê de Ética em Pesquisa (CEP) do Hospital de Clínicas de Porto Alegre, sob o número 130467.

A coleta de dados foi realizada na própria UBS onde os sujeitos do estudo atuam, durante o mês de dezembro de 2013, por meio de entrevistas semiestruturadas, gravadas em audiotape, textualizadas e, em seguida, transcritas para fins de análise e interpretação dos dados coletados. Os nomes dos sujeitos foram substituídos pela abreviatura $E$. (entrevistado), seguida de um número ordinal.

Os dados foram analisados por meio da análise de conteúdo da modalidade temática, operacionalmente, efetuada em três etapas: pré-análise, exploração do material e tratamento dos resultados obtidos e interpretação dos dados. Na pré-análise tomou-se contato com o material produzido na transcrição das entrevistas, por meio de leitura exaustiva, com vistas a uma impregnação das informações contidas; na exploração, realizou-se a categorização dos dados, organizando-se as unidades de registro a partir de suas afinidades temáticas; e, na interpretação buscou-se a compreensão e interpretação dos dados à luz do referencial (MINAYO, 2010).

\section{Resultados e discussão}

Quando questionados acerca de sua concepção sobre o processo de envelhecer, diferentes respostas e percepções emergiram dos depoimentos dos profissionais de saúde. Estas foram agrupadas nas seguintes categorias: processo natural do ser humano, autonomia e qualidade de vida, o jeito antigo e o novo, as perdas. 
Alguns dos entrevistados percebem o envelhecimento como um processo natural na vida do ser humano, marcado por mudanças fisiológicas, emocionais e sociais, vivenciadas de formas distintas, de acordo com a história, as experiências e as condições de vida que cada pessoa teve durante sua trajetória.

Envelhecer é uma fase, é fisiológico. É uma fase da vida que todo mundo vai passar [...] são as mudanças, as transformações que a gente vai tendo em decorrer da fase da vida (E. 4).

[...] é um processo natural, um processo do teu desenvolvimento. Faz parte da tua vida, o envelhecimento. Eu sempre brinco que quem não envelhece, morre antes. Eu acho que é uma fase que a gente tem alguns ganhos e algumas perdas, como em tudo na vida [...] (E. 11).

É um processo natural, desde que a gente nasce está passando [...] De forma geral, a gente está a envelhecer. Faz parte da vida, isso é natural. Envelhecer é bom (E. 13).

[...] tem várias formas pela qual a gente pode envelhecer [...] eu imagino que o envelhecimento seja uma fase como eu vou passar por todas [...] É uma fase que deveria ser encarada como fisiológica, encarada como qualquer outra. Sem a gente se apegar tanto aos aspectos negativos. Porque eu acho que coisas negativas nós temos em todas as fases da vida, não só no envelhecimento. Mas parece que quando a gente ouve falar da palavra envelhecimento, a gente logo se apega a limitações físicas, emocionais, aposentadoria [...] (E. 15).

A velhice é difícil de ser definida, principalmente quando se almeja uma velhice saudável, desejada por todos e para todos. Portanto, deve ser compreendida em sua totalidade e em suas múltiplas dimensões, visto que se constitui em um momento do processo biológico, mas não deixa de ser um fato social e cultural. Deve, ainda, ser entendida como uma etapa do curso da vida que, em decorrência do avançar da idade, ocorre modificações de ordem biopsicossocial que afetam as relações do indivíduo com o meio social ao qual está inserido (FREITAS; QUEIROZ; SOUSA, 2010).

Com o avançar da idade, ocorrem mudanças na vida da pessoa idosa, ocasionadas pelas condições de saúde ou pelo processo fisiológico do próprio envelhecimento. No entanto, é possível ter saúde e qualidade de vida na terceira idade, em que o idoso pode dispor do que a vida ainda proporciona, respeitando os limites do seu corpo, sem afastar a chance de fazer o que lhe seja prazeroso (GARBIN et al., 2010). Dessa forma, o processo de envelhecimento deve ser compreendido como um processo da vida. Envelhecemos porque vivemos e, muitas vezes, sem nos darmos conta disso (SOUZA; MATIAS; BRÊTAS, 2010). 
Alguns dos profissionais entrevistados relacionam a autonomia com a qualidade de vida no processo de envelhecer.

Eu acho que se 'tu' envelhecer com saúde [...] que possa ter uma vida, fazer o básico [...] eu acho que é a lei da vida. Tem que acontecer. Se essa pessoa tem essa qualidade, eu acho que é válido. Não em cima de uma cama, dependendo de tudo, aí é difícil. [...] a pessoa idosa sofre muito [...] Eu acho que é 'tu' ter uma qualidade, pelo menos, digna, principalmente de saúde (E. 2).

Envelhecer é ficar bem consigo mesmo, dependente da situação em relação à saúde, porque depende de cada pessoa a qualidade de vida. Acho que envelhecer é um fato da vida (E. 3).

Envelhecer é uma etapa. É mais uma etapa no processo da vida que deve ocorrer normalmente, mas com qualidade de vida, com autonomia (E. 8).

Envelhecer de forma ativa é sinônimo de vida plena e com qualidade. O envelhecimento com qualidade de vida equivale ao equilíbrio biopsicossocial e à integralidade do ser humano que se encontra inserido em um contexto social e que, mesmo sendo idoso, é capaz de desenvolver suas potencialidades (FERREIRA et al., 2010). Os conceitos saúde e qualidade de vida na velhice têm sido associados a questões de independência e autonomia, mas deve-se destacar que cada idoso sofre os "efeitos da idade" de diferentes maneiras (FERRETI; NIEROTKA; SILVA, 2011).

Entretanto, para conceituar qualidade de vida devem ser considerados os diversos olhares acerca de seu significado, pois este varia de indivíduo para indivíduo. Sua definição engloba diferentes representações, refletindo conhecimentos, experiências e valores dos indivíduos e da coletividade, que se reportam em épocas, espaços e histórias distintas, caracterizando-se como uma construção social. É importante que a equipe de saúde valorize a percepção do idoso sobre o significado de ter qualidade de vida para ele. Ninguém melhor que o próprio idoso para avaliar o que é mais importante e significativo em sua vida (RODRIGUES; LARA, 2011).

Nos depoimentos que seguem, as profissionais de saúde falam da mudança no pensamento de alguns anos atrás para os dias de hoje, em relação às questões ligadas ao envelhecimento. Antigamente, as pessoas idosas eram vistas como improdutivas e dependentes, sem autonomia para realizar seu autocuidado e suas atividades de vida. Hoje, os idosos continuam ativos em meio à sociedade. A idade deixou de ser um parâmetro que determina o que o idoso pode ou não fazer.

Também é mencionada a questão de que, nos dias atuais, as pessoas não dispõem de tempo para realizar determinadas atividades, como viajar, dançar, 
sair e conversar com amigos, em razão do trabalho e dos compromissos que assumem ao longo da vida, adiando tais atividades para quando se aposentam e já tenham cumprido seu papel no trabalho e na criação dos filhos.

Hoje em dia, essa questão de envelhecer está bem diferente do que era antigamente. Antigamente, as pessoas envelheciam e deixavam de fazer várias coisas, ficavam esperando a morte chegar. E agora não. A concepção que eu tenho de envelhecer agora é que as pessoas são bem ativas. Hoje em dia a gente vê idosas de 80 anos, caminhando, fazendo exercício [...] Eu pretendo envelhecer assim, bem ativa, poder viajar bastante, aproveitar [...] (E. 6).

Eu pretendo envelhecer muito bem. Eu pretendo fazer muitas coisas que eu tenho vontade de fazer hoje e não faço por não ter tempo [...] eu pretendo me integrar a grupos, viajar, passear, conhecer gente nova, dançar, cantar, fazer ginástica [...] Eu pretendo fazer bastante coisa que eu não faço agora, por ser eu e os filhos [...] Pretendo fazer coisas pra mim, pra mim me alegrar, pra mim viver bem, envelhecer bem, com alegria e na companhia de amigos, conhecidos e familiares (E. 7).

[...] eu acho que o processo de envelhecimento é uma experiência de aprendizado, de lidar com limitações, de aprender a ter outras formas de lazer, de se sentir bem consigo, mesmo com outras atividades, muitas vezes, em função de limitações físicas [...] eu vejo como um aprendizado, cada um com seu processo [...] uma situação saudável como um todo (E. 14).

Para Ferreira et al. (2009), a velhice é uma época em que as tarefas básicas em relação ao desempenho profissional e à família, de certo modo, foram cumpridas e o indivíduo pode se sentir livre para realizar seus desejos e sonhos.

A ideia do envelhecimento associado a doenças nem sempre se mostra como uma realidade na vida do idoso. Mesmo tendo de conviver com algumas perdas, o desenvolvimento de atividades, o engajamento familiar e o incentivo à participação social podem favorecer o envelhecimento saudável (CIOSAK et al., 2011).

Mesmo com as limitações que podem ocorrer com o passar dos anos, o idoso pode redescobrir alternativas de viver sua própria vida com qualidade. Tal possibilidade aumentará no momento em que a sociedade considerar a importância do contexto familiar e social na vida do idoso, além de reconhecer suas potencialidades e o valor das pessoas idosas (CIOSAK et al., 2011).

Portanto, considerar a mudança de conceitos que a sociedade vem sofrendo acerca do envelhecimento é uma maneira de se envelhecer bem e com saúde, alegria e bem-estar, deixando de lado os paradigmas que remetem o envelhecimento somente a doenças, perdas, limitações e sofrimento (GARBIN et al., 2010). 
Muitos profissionais descreveram o processo de envelhecimento como uma fase natural da vida, permeado por mudanças fisiológicas que podem dificultar a vida do idoso, mas que não o impedem de desfrutar de um envelhecimento saudável. Alguns dos profissionais entrevistados, entretanto, mencionam o envelhecimento como uma fase carregada de perdas, tanto físicas quanto emocionais, sendo um processo difícil na vida das pessoas, em detrimento de todas as incapacidades e limitações que muitos apresentam com o avançar da idade. Outra questão pontuada pelos profissionais é a ideia de que o envelhecer em si já traz consigo o pensamento de morte na vida dos idosos, conforme se observa nos depoimentos a seguir.

Eu acho que o envelhecer é um repensar de toda tua vida. É um momento que a gente começa a pensar na morte, também. O envelhecer traz esse pensamento. E, às vezes, o envelhecer está ligado a alguma doença [...] (E. 5).

[...] Esse processo é bastante doloroso [...] Eu acho que é um momento de bastante maturidade das pessoas. Deveria ser um momento que, emocionalmente, as pessoas deveriam sentir-se mais estáveis, mas não é o que a gente vê na prática. A gente vê as pessoas bastante afetadas, pelo aspecto econômico, aspecto familiar, abandono [...] (E. 9).

Envelhecer, além do avanço da idade, significa perda de funções, diminuição de capacidades, maior propensão a doenças, maior vulnerabilidade (E. 10).

Eu acho que o processo de envelhecimento causa toda uma modificação nas funções orgânicas, uma diminuição na questão da mobilidade e um pouco da cognição, mas tudo de uma forma que não chega a afetar a saúde da pessoa idosa [...] Mas o que estiver incomodando ou causando prejuízo não é o normal (E. 12).

O envelhecimento faz parte do processo de desenvolvimento do ser humano, que depende, dentre outros aspectos, da história individual de cada pessoa, das dificuldades enfrentadas, do contexto que está inserido, da rede de apoio familiar e social e da forma como cada um compreende o envelhecimento e a velhice (GUERRA; CALDAS, 2010).

No entanto, o que se observa é que, quando se referem às vivências frente ao processo de envelhecimento, as pessoas frequentemente fazem associações de natureza predominantemente negativas, destacando a insuficiência, incapacidade, limitação, perda, vulnerabilidade, dependência, solidão e abandono. $\mathrm{O}$ reconhecimento da velhice relacionada às perdas e ao declínio das habilidades do idoso esquece que o desenvolvimento humano é um processo compensatório entre perdas e ganhos. Os idosos precisam superar suas transformações 
e os preconceitos impostos pela sociedade (LIMA, 2006). Essa visão negativa do envelhecimento que muitos compartilham, acaba gerando situações que impossibilitam as oportunidades do idoso desfrutar de uma velhice saudável (SILVA; FOSSATTI; PORTELA, 2007).

Na velhice, o ser humano fica mais sujeito às perdas evolutivas em seus diversos domínios, em virtude de eventos biológicos, psicológicos e sociais, característicos de sua história individual e acontecimentos que ocorrem ao longo do curso da história de cada sociedade. Isso não significa que na velhice acontecem mais perdas do que ganhos, não quer dizer que a velhice é sinônimo de doença, ou que as pessoas ficam impedidas de se envolverem com outras atividades. Viver significa adaptação ou possibilidade de constante autorregulação, tanto em termos biológicos quanto em termos psicológicos e sociais (FREITAS; QUEIROZ; SOUSA, 2010).

\section{Conclusão}

Este estudo surgiu com a proposta de conhecer a visão que os profissionais de saúde têm sobre o processo de envelhecimento, visto que estes trabalham diretamente na assistência à saúde da pessoa idosa. Pensar nas representações do envelhecimento pode auxiliar a compreensão do profissional de saúde diante desse processo. Para atender essa nova demanda e os novos paradigmas acerca do envelhecer, torna-se imprescindível uma assistência à saúde que contemple a promoção da saúde e prevenção de agravos à saúde do idoso, percebendo-o em sua totalidade, diante de seu contexto biológico, psicológico, familiar, social e espiritual.

A forma de olhar o idoso como um ser ativo, participante e com autonomia para decidir sobre sua vida têm modificado as ações de saúde direcionadas a essa população. Hoje, é essencial manter os idosos socialmente ativos na comunidade em que vivem e os profissionais da Estratégia de Saúde da Família têm contribuído de forma significativa para isso.

Envolver o idoso em seu cuidado e valorizar seu conhecimento e sabedoria adquiridos durante a vida é uma forma de qualificar a assistência a essa faixa etária. Há a necessidade de compreender o idoso e interagir com ele para que haja resolutividade nas ações realizadas pela equipe de saúde. Quando as ações são planejadas e organizadas a partir das necessidades relatadas pelo próprio idoso, estas apresentam melhores resultados no processo de cuidar e ser cuidado. 
Com base nestes pressupostos, esperamos que o estudo possa contribuir para a melhoria da prática assistencial prestada ao idoso. Para tanto, parte dos profissionais de saúde o interesse por um aprendizado contínuo, a fim de embasar e fortalecer o conhecimento teórico-prático sobre os cuidados destinados ao idoso. É importante capacitar o profissional para o reconhecimento das necessidades de saúde da pessoa idosa, respeitando suas limitações e potencialidades.

Ainda, mudanças de conceitos e paradigmas são necessárias para que haja uma produção de saúde que contemple a pessoa idosa em todas suas dimensões, visto que se trata de um grupo etário com características bastante peculiares que devem ser consideradas pelos profissionais e serviços de saúde que prestam assistência a essa parcela da população.

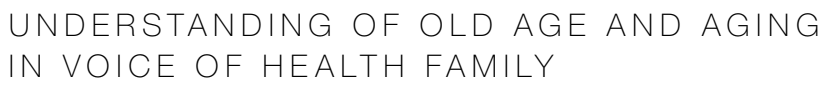

Ageing is a complex process of change in the trajectory of life. Reflect on representation of aging is a way to understand the real meaning of aging, allowing health professionals planning strategies grounded in reality. The objective is to know what working in the Family Health Strategy (FHS) professionals attribute to aging and their perception of this process. This is part of a research entitled "Care for the elderly in view of professionals of the Family Health Strategy." The research is a descriptive study of a qualitative nature, designed with professionals FHS a Basic Health Unit in Porto Alegre/RS. The sample was randomly selected and consisted of 16 professionals (four doctors, four nurses, four nursing technicians and four community health workers). Data collection occurred in December 2013, through semi-structured interviews. The analysis of the data was by thematic content analysis proposed by Minayo. When asked about his views on the process of aging, different perceptions emerged, grouped in categories: natural process of the human being, autonomy and quality of life, the old and the new way, the losses. Aging brings changes to society. To meet this demand and paradigms about aging is that professionals need to meet the elderly in their entirety. There is a need to understand the elderly and interact with it so there solving the actions taken by the health team. 
Family health. Health of the elderly. Aging. Health professional.

referências

CIOSAK, Suely Itsuko et al. Senescência e senilidade: novo paradigma na atenção básica de saúde. Revista da Escola de Enfermagem USP, São Paulo, v. 45, n. especial 2 , p. 1763-1768, dez. 2011. Disponível em: <http://www.scielo.br/pdf/reeusp/v45nspe2/22. pdf>. Acesso em: 14 jul. 2014

FERREIRA, Aleksandro Belo et al. Programa de atenção particularizada ao idoso em unidades básicas de saúde. Saúde e Sociedade, São Paulo, v. 18, n. 4, p. 776-786, maio. 2009. Disponível em: <http://www.scielo.br/pdf/sausoc/v18n4/20.pdf>. Acesso em: 16 maio 2013.

FERREIRA, Olívia Galvão Lucena et al. O envelhecimento ativo sob o olhar de idosos funcionalmente independentes. Revista da Escola de Enfermagem USP, São Paulo, v. 44, n. 4, p. 1065-1069, dez. 2010. Disponível em: <http://www.scielo.br/pdf/reeusp/ v44n4/30.pdf>. Acesso em: 12 jul. 2014

FERRETTI, Fátima; NIEROTKA, Rosane Paula; SILVA, Márcia Regina da. Concepção de saúde segundo relato de idosos residentes em ambiente urbano. Interface - Comunicação, Saúde, Educação, Botucatu, v. 15, n. 37, p. 565-572, 2011.

FREITAS, Maria Célia de; QUEIROZ, Terezinha Almeida; SOUSA, Jacy Aurélia Vieira de. O significado da velhice e da experiência de envelhecer para os idosos. Revista da Escola de Enfermagem USP, São Paulo, v. 44, n. 2, p. 407-412, jun. 2010. Disponível em: <http://www.scielo.br/pdf/reeusp/v44n2/24.pdf>. Acesso em: 16 maio 2013

GARBIN, Cléa Adas Saliba et al. O envelhecimento na perspectiva do cuidador de idosos. Ciência e Saúde Coletiva, Rio de Janeiro, v. 15, n. 6, p. 2941-2948, set. 2010 Disponível em: <http://www.scielo.br/pdf/csc/v15n6/a32v15n6.pdf>. Acesso em: 12 jul. 2014.

GUERRA, Ana Carolina Lima Cavaletti; CALDAS, Célia Pereira. Dificuldades e recompensas no processo de envelhecimento: a percepção do sujeito idoso. Ciência e Saúde Coletiva, Rio de Janeiro, v. 15, n. 6, p. 2931-2940, set. 2010. Disponível em: <http:// www.scielo.br/pdf/csc/v15n6/a31v15n6.pdf>. Acesso em: 12 jul. 2014.

HAMMERSCHMIDT, Karina Silveira de Almeida; LENARDT, Maria Helena. Tecnologia educacional inovadora para o empoderamento junto a idosos com diabetes mellitus. Texto Contexto Enfermagem, Florianópolis, v. 19, n. 2, p. 358-365, abr./ jun. 2010. Disponível em: <http://www.scielo.br/pdf/tce/v19n2/18.pdf>. Acesso em: 21 maio 2014.

LIMA, Leda Hecker Pereira. Eu e o envelhecimento. Porto Alegre: AGE, 2006.

MINAYO, Maria Cecília de Souza. O desafio do conhecimento: pesquisa qualitativa em saúde. 12. ed. São Paulo: Hucitec, 2010

RODRIGUES, Ana Cristina Coelho; LARA, Maristela Oliveira. Qualidade de vida do idoso: um levantamento da produção científica nos últimos dez anos. Revista de Enfermagem do Centro-Oeste Mineiro, São João Del Rei, v. 1, n. 3, p. 395-406, jul./ set. 2011. 
SILVA, Carine Alves da; FOSSATTI, Anderlei Fabiano; PORTELA, Marilene Rodrigues. Percepção do homem idoso em relação às transformações decorrentes do processo de envelhecimento humano. Estudos Interdisciplinares sobre o Envelhecimento, Porto Alegre, v. 12, p. 111-126, 2007.

SOUZA, Rosangela Ferreira de; MATIAS, Hernani Aparecido; BRÊTAS, Ana Cristina Passarella. Reflexões sobre envelhecimento e trabalho. Ciência e Saúde Coletiva, Rio de Janeiro, v. 15, n. 6, p. 2835-2843, set. 2010. Disponível em: <http://www.scielo. br/pdf/csc/v15n6/a21v15n6.pdf>. Acesso em: 08 jun. 2013.

Recebido em: 11/06/2014

Aceite final em: 11/12/2014 